\title{
Neurotransmission of the Bezold-Jarisch reflex in the nucleus tractus solitarii of sino-aortic deafferentated rats
}

\author{
Deoclécio A. Chianca Jr. ${ }^{\text {a }}$, Leni G.H. Bonagamba ${ }^{\text {b }}$, Benedito H. Machado ${ }^{\text {b, * }}$ \\ ${ }^{\text {a }}$ Department of Biological Sciences, Federal University of Ouro Preto, Ouro Preto, MG, Brazil \\ ${ }^{\mathrm{b}}$ Department of Physiology, School of Medicine of Ribeirão Preto, University of São Paulo, 14049-900, Ribeirão Preto, SP, Brazil
}

Accepted 7 January 1997

\begin{abstract}
The Bezold-Jarisch (B-J) reflex was activated by serotonin (5-HT, i.v.) before and $10 \mathrm{~min}$ after bilateral microinjection of increasing doses of kynurenic acid, a non-selective antagonist of excitatory amino acid (EAA) receptors, into the commissural nucleus tractus solitarii (NTS) of sino-aortic deafferentated (SAD) and sham-operated (SO) unanesthetized rats. Increasing doses of kynurenic acid produced a dose-dependent blockade of the bradycardic and hypotensive responses to B-J reflex activation in both SO (from 0.1 to 10.0 $\mathrm{nmol} / 100 \mathrm{nl}$ ) and SAD (from 0.1 to $2.0 \mathrm{nmol} / 100 \mathrm{nl}$ ). Comparison of the effect of kynurenic acid on the hypotension and bradycardic dose-response curves showed a significant difference between SO and SAD rats, indicating that smaller doses of kynurenic acid are required in SAD rats than in SO rats to block the neurotransmission of the B-J reflex in the NTS. The data also showed that bilateral microinjection of kynurenic acid into the NTS at doses of $0.5(131 \pm 7$ vs. $115 \pm 8 \mathrm{mmHg})$ and $2.0 \mathrm{nmol} / 100 \mathrm{nl}(140 \pm 11 \mathrm{vs.} 116 \pm 9$ $\mathrm{mmHg}$ ) produced an acute and significant increase in the basal mean arterial pressure of SAD rats similar to that observed with the same doses in SO rats, which was back to control values 5-10 min later. The increase in basal mean arterial pressure immediately after kynurenic acid microinjection into the NTS of SAD rats suggests that in the absence of the arterial baroreceptors, the B-J reflex plays an important role in the autonomic regulation of the circulation. The data also show different dose-response curves for hypotension and bradycardia in response to B-J reflex activation in SAD than in SO rats in the presence of increasing doses of kynurenic acid into the NTS, indicating that the neurotransmission of the B-J reflex in the NTS of SAD rats is more sensitive to the blockade of the EAA receptors than in SO rats.
\end{abstract}

Keywords: Afferent C-fiber; Cardiopulmonary receptor; Excitatory amino acid receptor; Neurogenic hypertension; Neurotransmission

\section{Introduction}

The physiological role of the cardiopulmonary afferent $\mathrm{C}$-fibers in the regulation of arterial pressure (AP) in normal animals is not completely understood $[12,15]$. Studies performed by Persson et al. [14] have shown that cardiopulmonary receptors play an important role in the regulation of AP after the removal of arterial baroreceptors in dogs. We have shown that the cardiovascular responses produced by the activation of the Bezold-Jarisch (B-J) reflex by serotonin (5-HT, i.v.) are significantly greater after sino-aortic deafferentation (SAD) than in sham-operated (SO) rats and also that the neurotransmission of the cardiovagal component of the B-J reflex in the NTS of

\footnotetext{
* Corresponding author. Fax: +55 (16) 633-0017. E-mail: bhmachad@fmrp.usp.br
}

normal rats is mediated by NMDA receptors. [3,4]. The data from our previous studies, taken together, suggest an important role for the cardiopulmonary receptors in circulatory control after removal of arterial baroreceptors and indicate an important role for the excitatory amino acid (EAA) receptors in the neurotransmission of the B-J reflex in the NTS. However, the characteristics of the neurotransmission of the B-J reflex in the NTS of SAD rats, particularly under unanesthetized conditions, has not been studied before.

The aim of the present study was to evaluate whether the neurotransmission of the B-J reflex in the NTS is altered after SAD. Thus, we microinjected kynurenic acid, a non-selective antagonist of EAA receptors, into the NTS of SO and SAD rats and examined the cardiovascular responses produced by activation of the B-J reflex and the changes in the basal MAP and HR of these animals. 


\section{Methods}

Male Wistar rats weighing 250-270 g were used in the present study. Four days before to the experiments, after a 12-h fast, the rats were submitted to pentobarbital sodium anesthesia (40 mg/kg, intraperitoneally (i.p.)) and later, under deep anesthesia, were placed in a stereotaxic apparatus (David Kopf Instruments, USA) and implanted with guide cannulas in the direction of the NTS, according to the coordinates of Paxinos and Watson [13] and using the technique of Michelini and Bonagamba [11]. The deep level of anesthesia was carefully followed during the stereotaxic surgery by frequently pinching the paw and additional anesthetic was injected ip when required. The guide cannulas (15 mm long) were introduced perpendicularly $0.5 \mathrm{~mm}$ lateral to the midline, $14 \mathrm{~mm}$ caudal to the bregma and $7.9 \mathrm{~mm}$ below the skull surface. The tip of the guide cannula was placed in the cerebellum $1.0 \mathrm{~mm}$ above the dorsal surface of the brain stem. The cannulas were fixed to the skull with methacrylate and watch screws and occluders were used until the time for microinjection. The needle used for microinjection (33 gauge, Small Parts, Inc., FL) into the NTS was $1.5 \mathrm{~mm}$ longer than the guide cannula and was connected by PE-10 tubing to a $1 \mu \mathrm{l}$ syringe (Hamilton, Reno, NV).

One day before the experiments one group of rats was submitted to $\mathrm{SAD}$, under ether anesthesia, by the technique of Krieger [8]. Another group of rats was submitted to sham operation (SO) and both groups, under the same anesthesia, had a catheter inserted into the femoral artery for measurement of pulsatile arterial pressure (PAP) and heart rate (HR) and a second catheter inserted into the femoral vein for serotonin (5-HT) and phenylephrine injection. Both catheters were tunneled and exteriorized through the back of the neck to be connected to the pressure transducer under conscious freely moving conditions. PAP and mean arterial pressure (MAP) were measured with a pressure transducer (COBE CDX III, COBE Laboratories, Inc., Lakewood, $\mathrm{CO}$ ) connected to a Narcotrace 80 Physiological Recorder (NARCO Bio-Systems, Austin, TX) and HR was quantified with a NARCO Biotachometer Coupler (Model 7302).

The efficacy of SAD was evaluated by intravenous injection of phenylephrine $(0.5 \mu \mathrm{g} / \mathrm{rat})$ before the experiments and only the rats that presented a bradycardia of less than $20 \mathrm{bpm}$ in response to an increase of $35-40 \mathrm{mmHg}$ in mean arterial pressure (MAP) were included in the SAD group. The B-J reflex was activated by the injection of 5 -HT at a dose $(8.0 \mu \mathrm{g} / \mathrm{kg}$, i.v. $)$ that produces significant hypotension and bradycardia $[3,4]$. The cardiovascular effects of 5-HT were effectively mediated by the vagus nerve efferent to the heart, considering that in previous studies we were able to block the bradycardic and hypotensive responses to B-J reflex activation with methylatropine, i.v. [4]. In SAD rats, which present high arterial pressure lability, the activation of the B-J reflex was performed during periods of minor apparent variability in MAP. The injection of 5-HT was performed before and 10 and 40 min after bilateral microinjection of kynurenic acid into the NTS. In all groups of rats the cardiovascular responses to B-J reflex activation were back to control values $40 \mathrm{~min}$ after bilateral microinjections of kynurenic acid into the NTS, showing the reversibility of the EAA receptor blockade.

The kynurenic acid microinjected into the NTS was diluted in saline $(\mathrm{pH}$ 7.0) and the microinjections were performed in a volume of $100 \mathrm{nl}$. The needles for microinjection were carefully inserted sequentially into both guide cannulas and the microinjections performed manually. Three different doses of kynurenic acid $(0.1,0.5$ and $2.0 \mathrm{nmol} / 100 \mathrm{nl}$ corresponding to 1,5 and 20 millimolar, respectively) were microinjected into the NTS of three groups of SAD rats and four doses $(0.1,0.5,2.0$ and 10.0 $\mathrm{nmol} / 100 \mathrm{nl}$ corresponding to $1,5,20$ and 100 millimolar, respectively) were microinjected into the NTS of four groups of SO rats. The rats of each group received only one dose of kynurenic acid. The dose of $10.0 \mathrm{nmol} / 100 \mathrm{nl}$ was also tentatively microinjected into the NTS of SAD rats but this dose produced dramatic changes in ventilation and for this reason this group of SAD rats was not considered in the data analysis.

The activation of the Bezold-Jarisch reflex was performed $10 \mathrm{~min}$ before (control) and $10 \mathrm{~min}$ after bilateral microinjection of kynurenic acid into the NTS. The different doses of kynurenic acid into the NTS produced maximal changes in baseline MAP and HR 1-3 min after microinjection and the values observed at this time were considered to be the changes in baseline produced by kynurenic acid (Figs. 1 and 2). However, $10 \mathrm{~min}$ after bilateral microinjection of kynurenic acid when the B-J

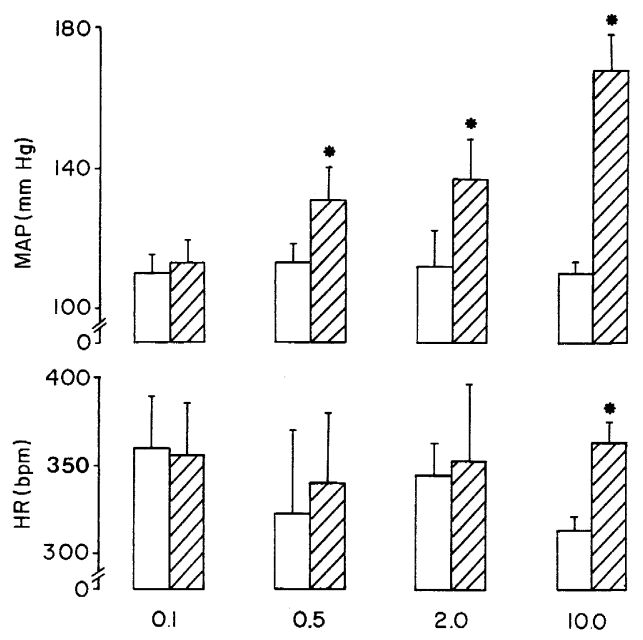

Fig. 1. Mean arterial pressure (MAP, upper panel) and heart rate (HR, lower panel) before (open bars) and 1-3 min after (hatched bars) bilateral microinjections of increasing doses of kynurenic acid $(0.1,0.5,2.0$ and 10.0 nmoles $/ 100 \mathrm{nl}$ ) into the NTS of four distinct groups of sham-operated rats $(* P<0.05)$. 


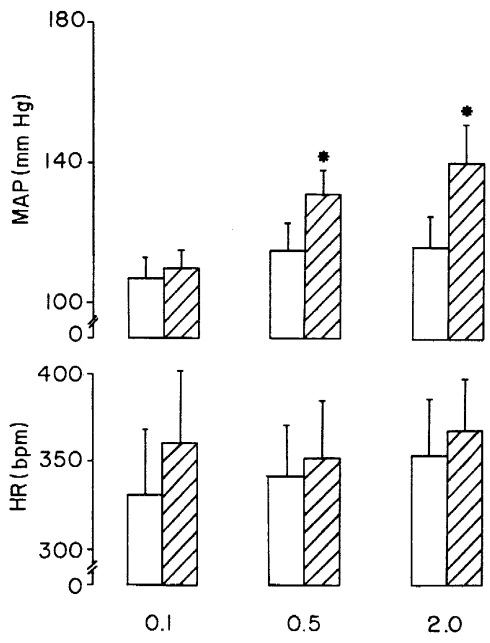

Fig. 2. Mean arterial pressure (MAP, upper panel) and heart rate (HR, lower panel) before (open bars) and 1-3 min after (hatched bars) bilateral microinjections of increasing doses of kynurenic acid (0.1, 0.5, and 2.0 $\mathrm{nmol} / 100 \mathrm{nl})$ into the NTS of three distinct groups of sino-aortic deafferentated rats $(* P<0.05)$.

reflex was activated again, MAP and HR were back to the control baseline values. In this case, the exception was the SO group that received the dose of $10 \mathrm{nmol} / 100 \mathrm{nl}$, in which the MAP remained significantly increased at that time.

After the experiments carried out under conscious conditions, $100 \mathrm{nl}$ of Evans blue (2\%) was microinjected into the same sites for histological analysis and later, under ether anesthesia, intracardiac perfusion with saline followed by $10 \%$ buffered formalin was performed. The brains were removed and 2 days later serial coronal sections $(10 \mu \mathrm{m})$ were cut and stained by the Nissl method. Only the rats with the microinjection sites located in the commissural NTS between the rostral and caudal edge of the area postrema were considered for data analysis. Statistical analysis for comparison between the dose response curves of SO and SAD rats was performed by analysis of variance (ANOVA) followed by the $F$-test for the differences between groups. The differences between changes in the basal MAP and HR before and after kynurenic acid were compared by the Student $t$-test and the level of significance was set at 0.05 in all cases.

\section{Results}

Fig. 1 summarizes the changes in basal MAP and HR observed 1-3 min after bilateral microinjection of increasing doses of kynurenic acid into the NTS of SO rats. The upper panel shows that the doses of $0.5(131 \pm 10$ vs. $113 \pm 5 \mathrm{mmHg}), 2.0(137 \pm 11$ vs. $112 \pm 4 \mathrm{mmHg})$ and $10.0 \mathrm{nmol} / 100 \mathrm{nl}(168 \pm 10$ vs. $110 \pm 3 \mathrm{mmHg})$ of kynurenic acid produced a significant increase in MAP
Table 1

Mean arterial pressure (MAP) and heart rate (HR) 10 min before and 10 min after bilateral microinjection of different doses of kynurenic acid $(0.1,0.5,2.0$ and $10.0 \mathrm{nmol} / 100 \mathrm{nl})$ into the NTS of sham-operated (SO) rats

\begin{tabular}{lllllll}
\hline Dose & \multicolumn{2}{l}{ MAP $(\mathrm{mmHg})$} & & \multicolumn{2}{l}{ HR $(\mathrm{bpm})$} & \multirow{2}{*}{$n$} \\
\cline { 2 - 3 } & Before & After & & Before & After & \\
\hline 0.1 & $102 \pm 3$ & $107 \pm 5$ & & $355 \pm 33$ & $350 \pm 27$ & 4 \\
0.5 & $114 \pm 4$ & $114 \pm 5$ & & $340 \pm 24$ & $337 \pm 19$ & 4 \\
1.0 & $120 \pm 4$ & $122 \pm 6$ & & $371 \pm 5$ & $377 \pm 11$ & 4 \\
10.0 & $107 \pm 5$ & $120 \pm 8\left({ }^{\mathrm{a}}\right)$ & & $380 \pm 29$ & $368 \pm 18$ & 6
\end{tabular}

${ }^{\mathrm{a}} P<0.05$

compared to control values. The lower panel shows that basal heart rate was significantly increased only after bilateral microinjection of $10.0 \mathrm{nmol} / 100 \mathrm{nl}$ of kynurenic acid into the NTS $(364 \pm 11$ vs. $314 \pm 7$ bpm). It is important to note that $10 \mathrm{~min}$ after bilateral microinjection of the doses of $0.1,0.5$ and $2.0 \mathrm{nmol} / 100 \mathrm{nl}$ of kynurenic acid into the NTS of SO rats, baseline MAP and HR were back to control baseline values (Table 1).

Table 1 presents the baseline values of MAP and HR 10 min before and $10 \mathrm{~min}$ after bilateral microinjection of different doses of kynurenic acid into the NTS of SO rats and indicates that only the MAP of the group that received the dose of $10 \mathrm{nmol} / 100 \mathrm{nl}$ remained significantly higher than control $10 \mathrm{~min}$ after the microinjection $(120 \pm 8$ vs. $107 \pm 5 \mathrm{mmHg}$ ).

Fig. 2 summarizes the changes in basal MAP and HR observed 1-3 min after bilateral microinjection of increasing doses of kynurenic acid into the NTS of SAD rats. The upper panel shows that the doses of $0.5(131 \pm 7$ vs. $115 \pm 8 \mathrm{mmHg})$ and $2.0(140 \pm 11$ vs. $116 \pm 9 \mathrm{mmHg})$ of kynurenic acid produced a significant increase in MAP compared to control values. The lower panel shows that basal HR was not significantly affected by the three different doses of kynurenic acid microinjected into the NTS of SAD rats. It is important to note that $10 \mathrm{~min}$. after bilateral microinjection of the doses of $0.1,0.5$ and $2.0 \mathrm{nmol} / 100$ $\mathrm{nl}$ of kynurenic acid into the NTS of SAD rats, baseline MAP and HR were back to control values (Table 2).

Table 2 presents the baseline values of MAP and HR 10 min before and $10 \mathrm{~min}$ after bilateral microinjection of

Table 2

Mean arterial pressure (MAP) and heart rate (HR) 10 min before and 10 min after bilateral microinjection of different doses of kynurenic acid $(0.1,0.5$ and $2.0 \mathrm{nmol} / 100 \mathrm{nl})$ into the NTS of sino-aortic deafferentated (SAD) rats

\begin{tabular}{|c|c|c|c|c|c|}
\hline \multirow[t]{2}{*}{ Dose } & \multicolumn{2}{|c|}{ MAP $(\mathrm{mmHg})$} & \multicolumn{2}{|c|}{ HR (bpm) } & \multirow[t]{2}{*}{$n$} \\
\hline & Before & After & Before & After & \\
\hline 0.1 & $127 \pm 11$ & $134 \pm 15$ & $376 \pm 40$ & $385 \pm 39$ & 5 \\
\hline 0.5 & $113 \pm 14$ & $103 \pm 8$ & $386 \pm 28$ & $348 \pm 12$ & 5 \\
\hline 2.0 & $110 \pm 15$ & $115 \pm 3$ & $398 \pm 42$ & $392 \pm 35$ & 5 \\
\hline
\end{tabular}


different doses of kynurenic acid into the NTS of SAD rats and indicates that the MAP of all groups was back to control baseline values $10 \mathrm{~min}$ after the microinjection.

Fig. 3 summarizes the bradycardic responses to B-J reflex activation by 5 -HT (i.v.) of SO and SAD rats before and $10 \mathrm{~min}$ after bilateral microinjections of increasing doses of kynurenic acid into the NTS. The data show that in SO rats (Fig. 3A) the doses of $0.1(-190 \pm 23$ vs. $-187 \pm 11 \mathrm{bpm}), 0.5(-162 \pm 31$ vs. $-160 \pm 34 \mathrm{bpm})$, $2.0(-205 \pm 15$ vs. $-127 \pm 30 \mathrm{bpm})$ and $10.0 \mathrm{nmol} / 100$ $\mathrm{nl}(-178 \pm 14$ vs. $-3 \pm 24 \mathrm{bpm})$ produced a dose dependent blockade of the bradycardic response to B-J reflex activation when compared with the responses of their respective controls. In SAD rats (panel B), the doses of 0.1 $(-166 \pm 25$ vs. $-150 \pm 42 \mathrm{bpm}), 0.5(-224 \pm 47$ vs. $-80 \pm 19 \mathrm{bpm})$ and $2.0 \mathrm{nmol} / 100 \mathrm{nl}(-212 \pm 25$ vs. $-28 \pm 9 \mathrm{bpm}$ ) also produced a dose-dependent blockade of the bradycardic response to B-J reflex activation. Statistical analysis (ANOVA) for comparison of the responses after kynurenic acid in the 0.1 to $2.0 \mathrm{nmol} / 100 \mathrm{nl}$ range showed a significant difference between these two doseresponse curves for SO and SAD rats.
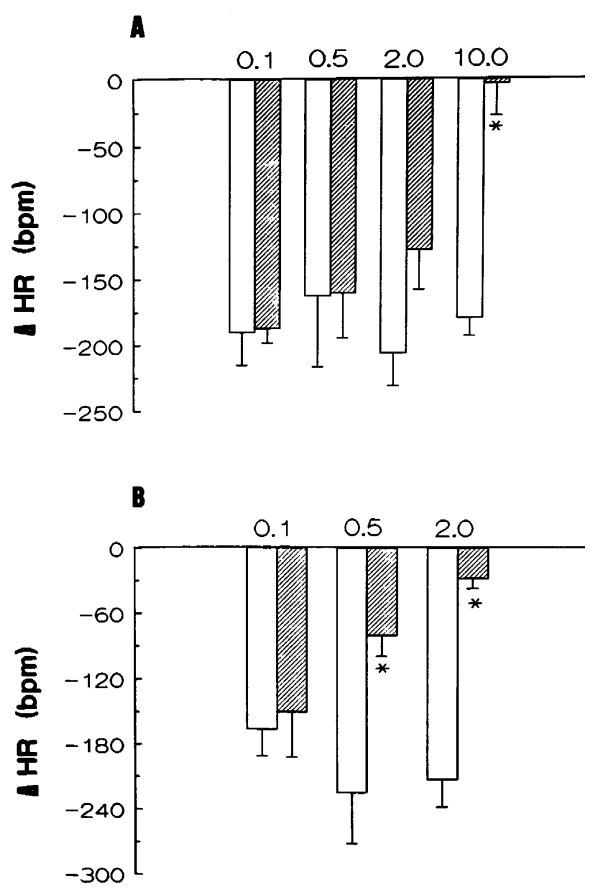

Fig. 3. A: bradycardic responses [changes in basal heart rate $(\Delta \mathrm{HR})$ ] to Bezold-Jarisch reflex activation by serotonin (5-HT, $8 \mu \mathrm{g} / \mathrm{kg}$, i.v.) before (open bars) and $10 \mathrm{~min}$ after (hatched bars) bilateral microinjections of kynurenic acid into the NTS at doses of 0.1, 0.5, 2.0 and 10.0 $\mathrm{nmol} / 100 \mathrm{nl}$ in four distinct groups of SO rats. B: bradycardic responses (changes in basal heart rate $(\Delta \mathrm{HR})$ ) to Bezold-Jarisch reflex activation by serotonin (5-HT, $8 \mu \mathrm{g} / \mathrm{kg}$, i.v.) before (open bars) and $10 \mathrm{~min}$ after (hatched bars) bilateral microinjections of kynurenic acid into the NTS in the doses of $0.1,0.5,2.0 \mathrm{nmol} / 100 \mathrm{nl}$ in three distinct groups of SAD rats. Analysis of variance (ANOVA) shows significant differences between the dose-response curves of SAD and SO rats $(P<0.05) .\left({ }^{*}\right)$ Different compared to the control response.

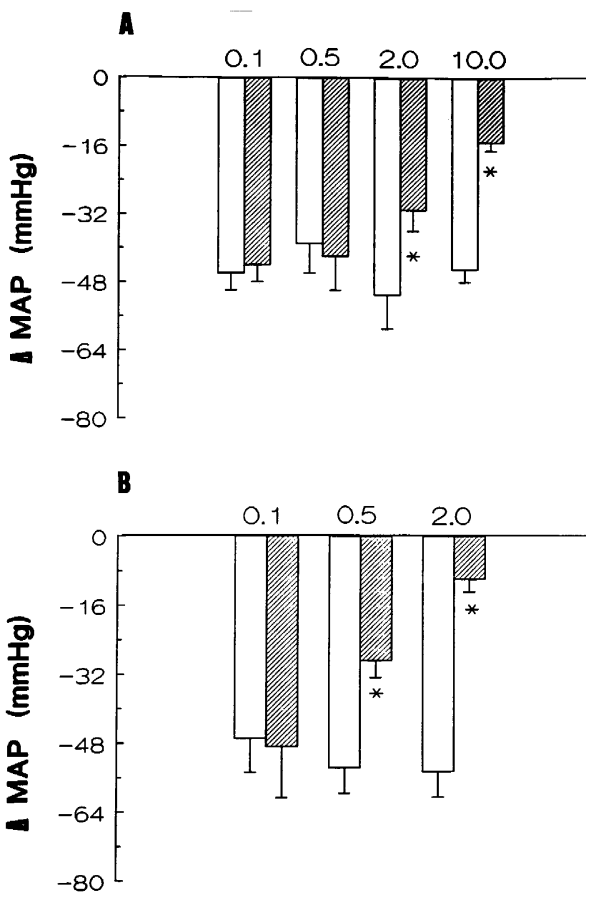

Fig. 4. A: hypotensive responses [changes in basal mean arterial pressure ( $\Delta$ MAP)] to Bezold-Jarisch reflex activation by serotonin (5-HT, 8 $\mu \mathrm{g} / \mathrm{kg}$, i.v.) before (open bars) and $10 \mathrm{~min}$ after bilateral (hatched bars) microinjections of kynurenic acid into the NTS at doses of 0.1, 0.5, 2.0 and 10.0 nmoles $/ 100 \mathrm{nl}$ in four distinct groups of SO rats. B: hypotensive responses [changes in basal mean arterial pressure ( $\Delta$ MAP)] to Bezold-Jarisch reflex activation by serotonin (5-HT, $8 \mu \mathrm{g} / \mathrm{kg}$, i.v.) before (open bars) and $10 \mathrm{~min}$ after bilateral (hatched bars) microinjections of kynurenic acid into the NTS at doses of $0.1,0.5$ and 2.0 nmoles / $100 \mathrm{nl}$ in three distinct groups of SAD rats. Analysis of variance (ANOVA) shows significant differences between the dose-response curves of SAD and SO rats $(P<0.05)$. ( $\left.{ }^{*}\right)$ Different compared to the control response.

Fig. 4 summarizes the hypotensive responses to B-J reflex activation by 5-HT (i.v.) of SO and SAD rats before and after bilateral microinjections of increasing doses of kynurenic acid into the NTS. In SO rats (Fig. 4A), the doses of $0.1(-46 \pm 4$ vs. $-44 \pm 4 \mathrm{mmHg}), 0.5(-39 \pm 7$ vs. $-42 \pm 8 \mathrm{mmHg}$ ), $2.0(-51 \pm 4$ vs. $-31 \pm 5 \mathrm{mmHg})$ and $10.0 \mathrm{nmol} / 100 \mathrm{nl}(-45 \pm 3$ vs. $-15 \pm 2 \mathrm{mmHg})$ produced a dose dependent blockade of the hypotensive response to B-J reflex activation when compared with their respective control responses. In SAD rats (Fig. 4B), the doses of $0.1(-47 \pm 8$ vs. $-49 \pm 12 \mathrm{mmHg}), 0.5(-54$ \pm 6 vs. $-29 \pm 4 \mathrm{mmHg})$ and $2.0 \mathrm{nmol} / 100 \mathrm{nl}(-55 \pm 6$ vs. $-10 \pm 3 \mathrm{mmHg}$ ) also produced a dose-dependent blockade of the hypotensive response to Bezold-Jarisch reflex activation. Statistical analysis (ANOVA) for comparison of the responses after kynurenic acid in the 0.1 to $2.0 \mathrm{nmol} / 100 \mathrm{nl}$ range showed a significant difference between the dose-response curves for SO and SAD rats.

Fig. 5 is a photomicrograph of a coronal section of the brainstem showing the sites of microinjection into the commissural NTS at the level of the rostral edge of the 


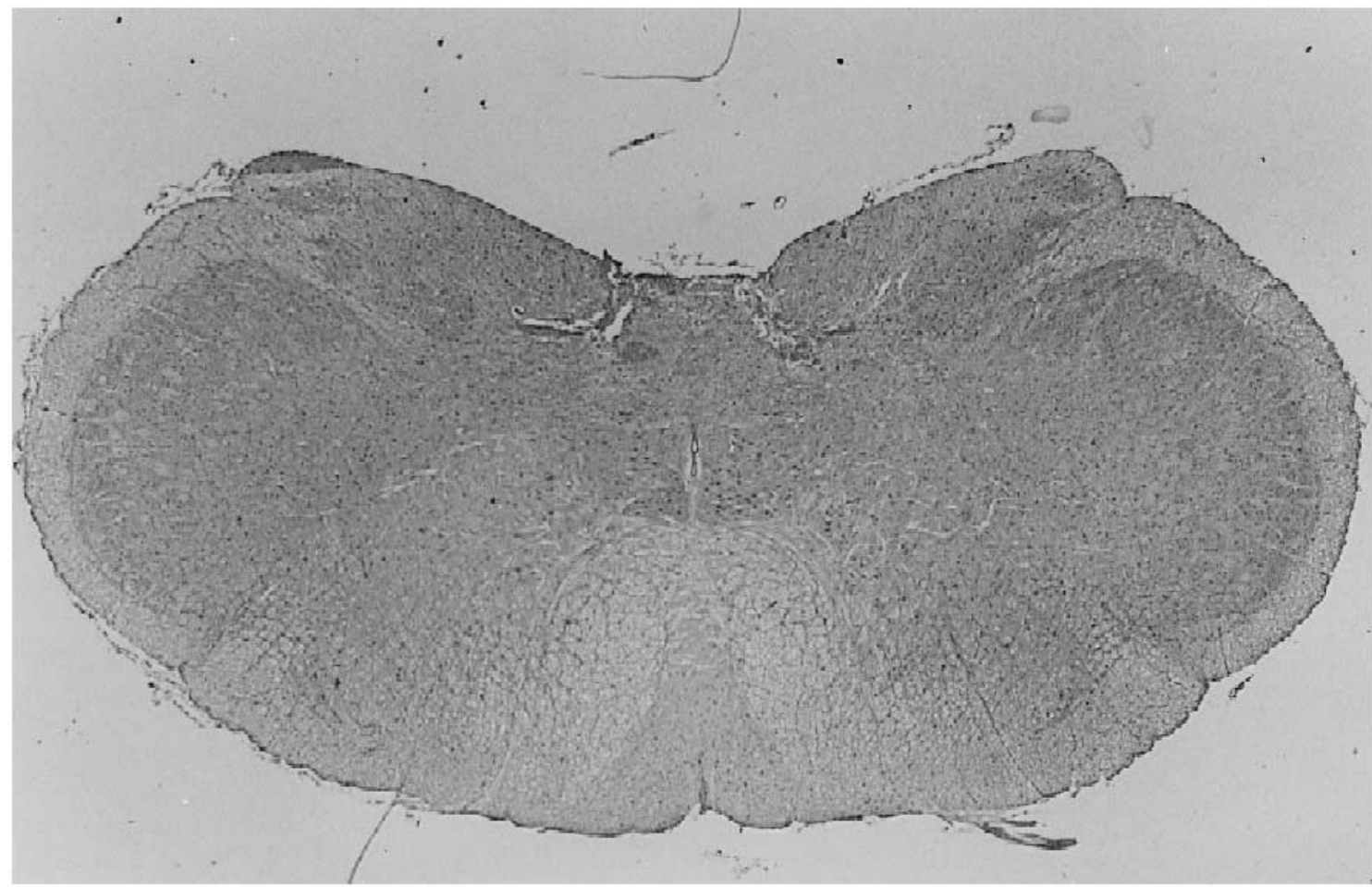

Fig. 5. Photomicrograph of a coronal section of the brain stem of one rat representative of the groups studied showing the sites of microinjection in the lateral portion of the commissural NTS at the level of the rostral edge of the area postrema.

area postrema of one rat representative of all groups studied.

\section{Discussion}

The removal of aortic and carotid baroreceptors produces an important imbalance in the autonomic regulation of blood pressure with a marked increase in arterial pressure lability, but no maintained hypertension has been observed in dogs and rats after deafferentation $[1,5,7,9,10]$. The unexpected absence of hypertension in SAD rats is an intriguing question that has been addressed by different studies [1-3,7,9]. In a recent study [3] we observed that the activation of the B-J reflex 1 and 15 days after SAD produced greater depressor and bradycardic responses than in SO rats and we suggested that SAD rats present increased sensitivity of the B-J reflex. These possibility was in accordance with studies by Persson et al. [14] showing that surgical removal of cardiopulmonary afferent C-fibers after SAD produces a significant increase in MAP in dogs. The increased sensitivity of the B-J reflex observed in our previous study could be explained by a possible change in neurotransmission at the NTS level, considering that studies by Vardhan et al. [17] and Verberne and Guyenet [18] demonstrated that the first synapse of the afferent C-fibers occurs at this level and is mediated by EAA receptors. In a recent study performed on unanesthetized rats we demonstrated that the neurotransmission of the cardiovagal component of the B-J reflex in the NTS is mediated by NMDA receptors [4].

In the present study we evaluated the possibility that the increased sensitivity of the B-J reflex could be due to changes in neurotransmission at the NTS level, where the cardiopulmonary, baro- and chemoreflex have their first synapse in the central nervous system. All of these 3 afferent systems seem to use L-glutamate as a neurotransmitter [6,16-18]. Therefore, in the absence of the arterial baroreceptor afferents in SAD rats, the neurotransmission of the B-J reflex may be modified, with a consequent change in the responses to the activation of this afferent system. The data obtained in the present study are consistent with this hypothesis, considering that kynurenic acid, a non-selective EAA receptor antagonist, produced a greater dose-dependent blockade of the hypotension and bradycardic response to the B-J reflex activation in SAD rats than in SO rats, probably because a smaller amount of the neurotransmitter is released at the NTS level. In addition, it is possible that the activation of cardiopulmonary receptors and baroreceptors produces cardiovagal excitation and sympatho-inhibition by activation of the same pool of EAA receptors in the post-synaptic neurons in the NTS. In this case, it is also possible that the absence of baroreceptor afferents to the NTS reduces the release of 
the neurotransmitter, with a possible increase of post-synaptic receptors sensitivity to the EAA receptor involved in the neurotransmission of the B-J reflex.

The activation of sympatho-inhibitory pathways by the cardiopulmonary afferents may modulate the basal sympathetic outflow keeping the MAP within a normal range considering that SAD rats are not hypertensive. This possibility is supported by the significant acute increase of MAP observed after bilateral microinjections of kynurenic acid into the NTS of SAD similar to the increase observed in SO rats. This increase in MAP after injection of kynurenic acid into the NTS of SAD rats, associated with the greater dose-dependent blockade of the B-J reflex by kynurenic acid in these rats, indicates that the neurotransmission of the B-J reflex at the NTS level is altered and suggests that this afferent system plays an important role in the cardiovascular regulation after removal of arterial baroreceptors.

No significant increase in HR was observed after kynurenic acid in the NTS of SAD rats, probably because basal HR was already increased due to the absence of arterial baroreceptors, while in SO rats a significant tachycardic response was observed. These differences in the changes in the basal HR and MAP between SO and SAD rats also suggest a dissociation of the autonomic processing of heart rate and vascular resistance at the NTS level and agree with our previous study showing that the neurotransmission of the cardiovagal component of the BezoldJarisch reflex is mediated by NMDA receptors [4]. Therefore, considering that the increase in the basal MAP of both SAD and SO rats after bilateral microinjection of kynurenic into the NTS and that the bilateral microinjection of AP-5, a selective NMDA receptor antagonist, produced no effect on baseline MAP in control rats [4], we may suggest that the sympatho-inhibitory component of the B-J reflex is mediated by non-NMDA receptors. However, additional studies are required to confirm this possibility.

We conclude that the neurotransmission of the B-J reflex in the NTS of SAD rats is more sensitive to the antagonism of EAA receptors and the findings that the basal MAP of SAD rats was significantly increased after kynurenic acid support the hypothesis that this reflex plays an important role in the autonomic regulation of the circulation after removal of the arterial baroreceptors.

\section{Acknowledgements}

The authors thank Rubens Fazan Jr. for the statistical analyses and Rubens F. Melo for the histological preparations. This work was supported by Fundação de Amparo à
Pesquisa do Estado de São Paulo (FAPESP, 91-0576-9) and Conselho Nacional de Desenvolvimento Científico e Tecnológico (CNPQ, 520.978/93-5).

\section{References}

[1] Alper, R.H., Jacob, H.J., Brody, M.J., Regulation of arterial pressure lability folowing sino-aortic deafferentation in rats, Am. J. Physiol., 253 (1987) H466-H476.

[2] Barres, C., Lewis, S.J., Jacob, H.J. and Brody, M.J., Arterial pressure lability and renal sympathetic nerve activity are dissociate in SAD rats, Am. J. Physiol., 32 (1992) R639-R646.

[3] Chianca-Jr., D.A. and Machado, B.H., The sensitivity of the Bezold-Jarisch reflex is increased in rats with sinoaortic deafferentation, Braz. J. Med. Biol. Res., 27 (1994) 775-781.

[4] Chianca-Jr., D.A. and Machado, B.H., Microinjection of NMDA antagonist into the NTS of conscious rats blocks the Bezold-Jarisch reflex, Brain Res., 718 (1996) 185-188.

[5] Cowley, A.W., Liard, L.D. and Guyton, A.C., Role of baroreceptor reflex in daily control of arterial blood pressure and other variables in dogs, Circ. Res., 32 (1973) 564-576.

[6] Gordon, F.J. and Talman,W.T., Role of excitatory amino acids and their receptors in bulbospinal control function. In: Central Neural Mechanisms in Cardiovascular Regulation. Eds. G. Kunos and J. Ciriello. Birkhäuser, Boston pp. 209-225, 1992.

[7] Jacob, H.J., Barres, C.P., Machado, B.H., Brody, M.J., Studies on neural and humoral contributions to arterial pressure lability, Am. J. Med. Sci., 295 (1988) 341-345.

[8] Krieger, E.M., Neurogenic hypertension in the rat, Circ. Res., 15 (1964) 511-521.

[9] Machado, B.H., Arterial pressure response to adrenergic blockade in rats with sino-aortic denervation, Braz. J. Med. Biol. Res., 23 (1990) 343-353.

[10] Mauad, H., Glass, M.L. and Machado, BH., Effect of selective denervation of baroreceptors on pulmonary ventilation and arterial pressure lability in rat, Hypertension, 19 (Suppl. II) (1992) II 182-II 186.

[11] Michelini, L.C. and Bonagamba, L.G.H., Baroreceptor reflex modulation by vasopressin microinjected into the nucleus tractus solitarii of conscious rats, Hypertension, 11: (suppl. I) (1988) I 75-I 79.

[12] Oberg, B. and White, S., Circulatory effects of interruption and stimulation of cardiac vagal afferents, Acta Physiol. Scand., 80 (1970) 383-394.

[13] Paxinos, G. and Watson, C., The Rat Brain in Stereotaxic Coordinates, Academic Press, New York, 1982.

[14] Persson, P., Ehmke, H., Kirchheim, H., Seller, H., Effect of sinoaortic denervation in comparison to cardiopulmonary deafferentiation on long-term blood pressure in conscious dogs, Pflügers Arch., 411 (1988) 160-166.

[15] Thoren, P., Role of cardiac vagal C-fibers in cardiovascular control, Rev. Physiol. Bioch. Pharmacol., 86 (1979) 1-94.

[16] Vardhan, A., Kachroo, A. and Sapru, H.N., Excitatory amino acid receptors in the nucleus tractus solitarius mediate the responses to the stimulation of cardio-pulmonary vagal afferent $\mathrm{C}$ fiber endings, Brain Res., 618 (1993) 23-31.

[17] Vardhan, A., Kachroo, A. and Sapru, H.N., Excitatory amino acid receptors in commissural nucleus of the NTS mediate carotid chemoreceptor responses, Am. J. Physiol., 264 (1993) R41-R50.

[18] Verberne, J.M.A. and Guyenet, P.G., Medullary pathway of the Bezold-Jarisch reflex in the rat, Am. J. Physiol., 32 (1992) R1195R1202. 